\title{
COMPARATIVE ANALYSIS OF CURRENT DENSITY METERS OPERATING IN SPACE PLASMAS
}

\author{
V. Korepanov, F. Dudkin \\ Lviv Centre of Institute of Space Researches, 5-A Naukova str., 290601, Lviv, Ukraine
}

\begin{abstract}
For experimental investigations of wave processes in space plasma one of the main tasks is the determination of dispersion relations between the wave vector and the frequency. The frequency analysis of fluctuations of magnetic field and electric current density in plasma is very efficient in this case. The measurements of the magnetic field fluctuations usually are made by a variety of magnetometers using well developed methods. Unfortunately, up to the moment there are no reliable measurements of space current density although some attempts to do it with an instrument called "Split Langmuir Probe" were made. It is mainly because of high sensitivity of the sensor readings to photocurrent and/or to spacecraft potential. The spatial current density can be measured not only by split Langmuir probe, but also by a contactless probe of the type of Rogovski coil and by Faraday cup. The comparative analysis of such probes operation in near Earth plasma is very important for further development of investigations of solar - terrestrial interactions. This study will be done both by theoretical and practical ways. As a first stage the theoretical analysis of application areas and of threshold characteristics of the current density probes will be executed. At a second stage the spatial experiment VARIANT carrying such probes as a scientific payload will be fulfilled.
\end{abstract}

\section{INTRODUCTION}

For experimental investigations of wave processes in space plasma one of the main tasks is the determination of dispersion relations between the wave vector and the frequency. It can be shown that simultaneous measurements of magnetic field and current density fluctuations allow to determine experimentally wave vector components. The frequency analysis of fluctuations of magnetic field and electric current density in plasma is very efficient in this case. The measurements of the magnetic field fluctuations usually are made by a variety of magnetometers using well developed methods. Unfortunately, up to the moment there are no reliable measurements of space current density, although some more or less successful attempts were made (Bering et al, 1984; Vaisberg et al, 1989).

The three sensors which could be used for the measurement of this value are proposed. First of all it is well known «Faraday Cup» instrument, installed at some spacecrafts for plasma population investigation (Safrankova et al, 1995). Then so called «Rogovsky Coil» sensor is proposed as a second meter. It was never used before for spatial current investigations, although its parameters can suit for this (Krasnossel'skikh et al, 1996). At last the sensor «Split Langmuir Probe» is investigated as the instrument for current density study. The theory of its operation is developed and discussed below.

An experimental array comprising all three instruments operating in parallel is proposed to install on the board of the satellite. This instrumentation will be prepared in the frames of a joint international spatial experiment VARIANT (Alleyne et al., 1998). The experiment is planned to perform onboard of the Ukrainian remote sensing satellite SICH-1M, that will be launched in 2000 at the polar circular orbit with the inclination of around $83^{\circ}$ and altitude $670 \pm 30 \mathrm{~km}$. The scientific payload includes three mentioned instruments for registration of space current density and also the sensors for measurements of the electric and magnetic field fluctuations in the frequency range from $0.1 \mathrm{~Hz}$ to $40 \mathrm{kHz}$. These measurements are complementary with the current density ones and they are supposed to allow to make comparison between direct and implicit estimates of the distribution and fine structure of currents. The next goal of VARIANT experiment is the simultaneous observation of the fine structure of 
currents in space and ground-based measurement of the ionospheric convection in the $\mathrm{E}$ and $\mathrm{F}$ layers making use of the system of radars SuperDARN.

\section{THE IMPORTANCE OF CURRENT DENSITY INVESTIGATION}

When wave activity in space plasma is investigated, the estimation of dispersion relations for electromagnetic (EM) waves is of great importance. It can be shown that the simultaneous measurements of orthogonal components of magnetic field and spatial current density fluctuations can give the wave vector $\bar{k}$ values for a spectrum of plane waves, by which wave field in plasma reference frame can be represented.

Each spectral component of plane wave may be written in the next form:

$$
\stackrel{\rho}{A}=\overbrace{0} \exp (-j(k \rho-\omega t)), \quad A=H, J,
$$

where $H, J$ - magnetic field and electric current density correspondingly, $j=(-1)^{0,5}, \bar{r}$ - vector distance, $\omega$ - angular frequency, $\mathrm{t}$ - time.

Let substitute expression (1) in Maxwell's equation

$$
[\nabla \times H]=J
$$

( $J$ may be the sum of displacement and conductivity currents) and assume that the space derivative of magnetic field is very small:

$$
\partial H_{0 i} / \partial x_{l} \rightarrow 0
$$

where $i, l=1,2,3 ; H_{0 i}$ - amplitude of $i$ - magnetic field component, $x_{l}$ - component of coordinate system.

Then we can obtain from (1)-(3) the following equation

$$
\left[\begin{array}{l}
\rho \\
k \times H
\end{array}\right]=j J
$$

or in scalar form in Cartesian coordinates $x, y, z$

$$
\left.\begin{array}{l}
k_{y} H_{z}-k_{z} H_{y}=j J_{x}, \\
k_{z} H_{x}-k_{x} H_{z}=j J_{y}, \\
k_{x} H_{y}-k_{y} H_{x}=j J_{z} .
\end{array}\right\}
$$

This system of equations allows to determine the unknown values $k_{m}, m=x, y, z$ by the measured values $H_{m}, J_{m}$. The amount of components necessary to measure can be reduced to any two if to use the next Maxwell's equation:

$$
\nabla \times \tilde{B}=0,
$$

then from expressions (1)-(3) we obtain

$$
k \times H=0
$$

or in scalar form

$$
\sum_{n=1}^{3} k_{n} H_{n}=0
$$

This explanation gives strong evidence of the theoretical possibility of $\bar{k}$ vector direct estimation. It is possible to say that there can not be objections as to the possibility to measure magnetic field fluctuations with very high resolution (Korepanov and Berkman, 1997). Now it is necessary to analyze whether the metrological parameters of the known instruments allow to measure $J$ value with enough high precision and whether the erroneous factors do not hinder the results. This is the aim of the investigation. By this it is postulated that the errors introduced by following processing in electronic circuitry are negligible. Only the physical limitations of all examined methods will be studied. 


\section{FARADAY CUP}

A Faraday cup (FC) is the device for the direct electron or ion current measurements in space plasma. Such measurements were realized, in particular, on board of "Prognoz" satellites and in experiment "Interball" (Safrankova et al, 1995).

The FC allows to measure the space current density $J$ by correlation:

$$
J=I S^{-1} \text {, }
$$

where $I$ is the current that goes through FC entrance window, $S$ is the area of this window. The FC schematic drawing is presented on fig. 1 .

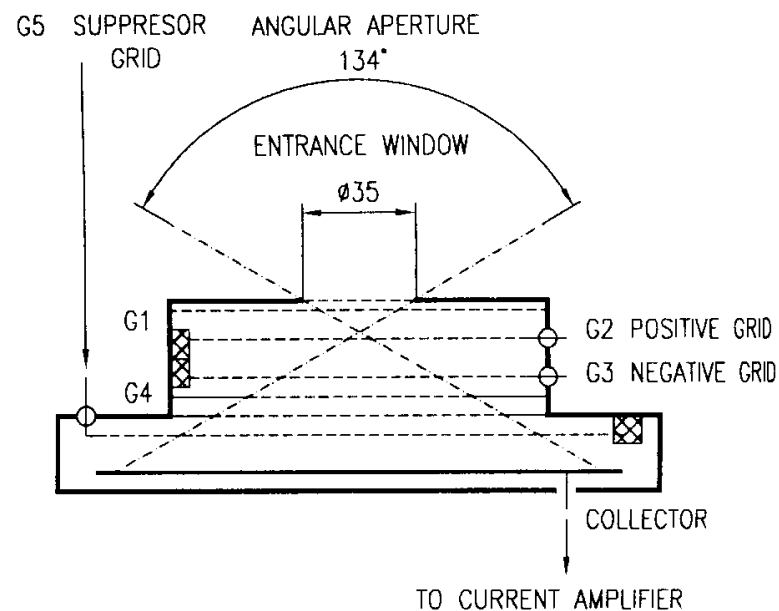

Fig.1.Faraday Cup Sensor diagram

The charged particles go through the entrance window (its area is about $10 \mathrm{sq} . \mathrm{cm}$ ) to collector. The current formed by these particles is transformed into voltage by current amplifier with high input resistance $R$ (about $10^{9}$ Ohms). Thus the input voltage is connected with current density by relation:

$$
u=J S R \text {. }
$$

The sign of collected particles depends on the sign of voltage on FC grids G2,G3. The entrance grid G1 protects the space near FC from influence of another grids potentials. The grid G4 protects in the same way the space near collector from influence of potentials of the grids G2,G3. The potentials of grids G1 and G4 have to be maintained to be equal to the potential of satellite. The suppressor grid G5 (the nearest to the collector) reduces the photoelectron and secondary electron currents from the collector. Additionally for reduction of the photoelectrons current the collector surface is corrugated and covered with a special black nickel coat. All these measures provide a photocurrent reduction in 200-300 times.

The space diagram pattern of FC is a circular cone with basement that oriented to FC. The angle at the top of the cone diametrical cross section or angular aperture is $134^{\circ}$ as shown on fig. 1 .

In order to provide the possibility of the measurement of charged particles fluxes along one axis it is necessary to have two of such FC's, pointing opposite sides. Then such arrangement will react to the charged particles fluxes or what is the same, spatial current flowing along given direction. The experimental model was constructed and the tests revealed enough good sensitivity of the device: about $10^{-11} \mathrm{~A} / \mathrm{cm}^{2}$ to current density fluctuations. As it is seen from this very short instrument description, from the first sight the main principal limitations of the method could be the influence of direct sunlight and high-energy charged particles and spacecraft floating potential changes. No other complications encountered with the relation (9) are expected.

\section{ROGOWSKY COIL}

The principle of Rogowsky Coil (RC) operation is based on the following concept. The instrument itself consists of the ring core from high permeability material with the toroidal winding on it (Fig. 2) (Krasnossel'skich et al, 1996). 


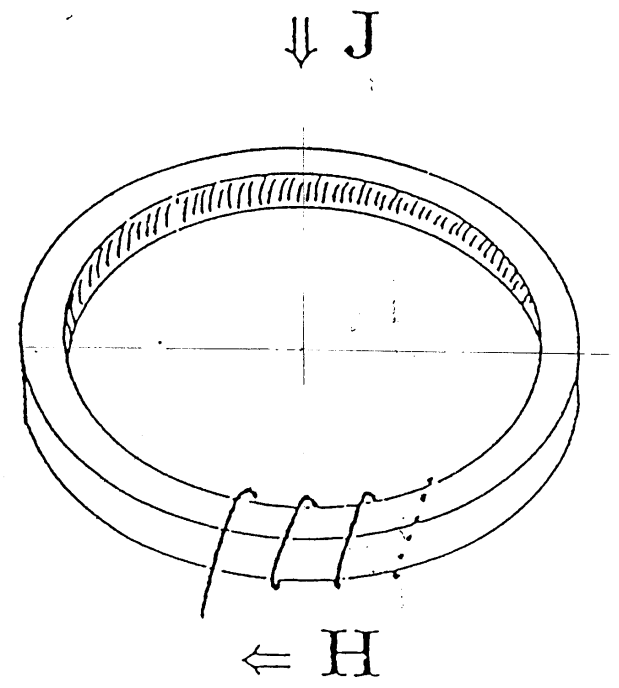

Fig.2. Rogowsky Coil sensor diagram

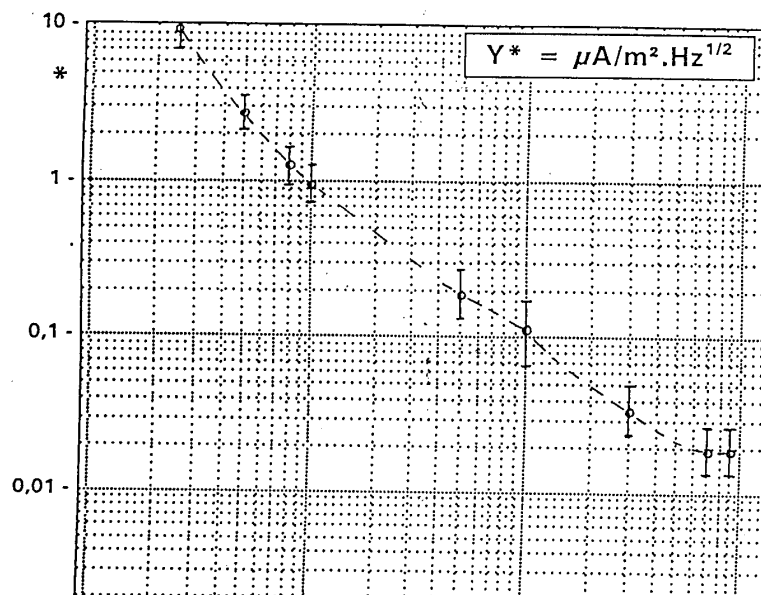

Fig.3. Rogowsky Coil noise level

The current $J$ flowing through the core inner hole in the direction parallel to the axis of this toroid causes magnetic field intercepting the turns of the RC winding. When the current $J$ is oscillating it provokes time varying magnetic field $\mathrm{H}$ and an e.m.f. $e_{\mathrm{i}}$ is induced in each turn of the winding:

$$
e_{i}=\mu S \frac{d H}{d t},
$$

where $S$ is core cross-section and $\mu$-permeability of the material.

The total e.m.f. $e$ consists of the summation of $e_{\mathrm{i}}(i=1, N)$, where $N$ is the number of turns that can be transformed into integral along the mean major radius $\rho$ of the toroid:

$$
e=\frac{1}{\Delta l} \sum_{i} e_{i} \Delta l=\frac{\mu S N}{2 \pi \rho} \frac{d}{d t} \oint H \cdot d l .
$$

According to Ampere's law, this integral may be expressed in term of the current flowing through the plane section of the core:

$$
\oint H \cdot d l=I_{\perp}=j_{\perp} \cdot \pi \rho^{2} .
$$

It is therefore seen that the output e.m.f. of the sensor depends upon the time derivative of the normal component of the current density by the relation

$$
e=\frac{\mu S N \rho}{2} \frac{d j}{d t}=K \frac{d j}{d t},
$$

where $K$ is the instrument's constant.

Such an instrument was constructed and showed rather good sensitivity having diameter $\rho$ equal to $30 \mathrm{~cm}$ : minimum noise level was about $10^{-12} \mathrm{~A} / \mathrm{cm}^{2} \cdot \mathrm{Hz}^{1 / 2}$ (Krasnossel'skikh et al, 1996). Namely the frequency dependence of the RC is its most serious principal limitation. The transfer function shape for the given device is presented on fig. 3, which reveals rather narrow frequency band of its application. But other specific noise sources, important for FC, especially influence of sunlight and high-energy charged particles, seem to be not distorting the $\mathrm{RC}$ operation.

\section{SPLIT LANGMUIRE PROBE}

The Split Langmuire Probe (SLP) operation principle is rather simple: two conducting plates are placed in space plasma at as small distance or split $d$ as possible and they are connected with a resistor $R_{\mathrm{S}}$ (Fig. 4). The SLP output signal $U$ is formed as follows:

$$
U=\mathrm{I} R_{\mathrm{s}}=\mathrm{J} S R_{\mathrm{s}}
$$

where $S$ is the surface of one SLP plate and $I$ - current via equivalent resistance of probe $R_{s}$. 
Further SLP is connected to the preamplifier and in order to investigate how it operates let us analyze its equivalent circuit (Fig. 5), where $R_{\mathrm{s}} C_{\mathrm{s}}$ are equivalent parameters of the sensor and $R_{\mathrm{i}}, C_{\mathrm{i}}$ - those of amplifier.

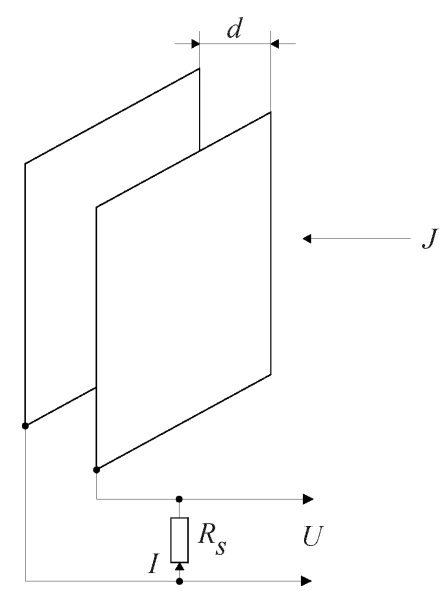

Fig.4. Split Langmuir Probe Diagram

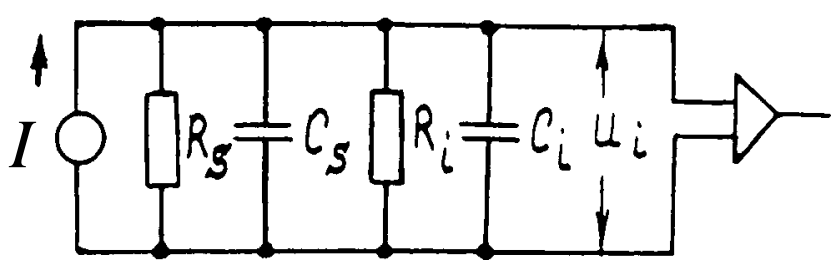

Fig.5. SLP equivalent diagram

The connection of $U$ with $J$ is investigated with following condition imposed:

$$
d \leq \lambda_{\mathrm{D}},
$$

where $\lambda_{\mathrm{D}}$ is Debye length.

The relation $U$ with $\mathrm{J}$ can be written as follows:

$$
U=J S R\left(1+(\omega \tau)^{2}\right)^{-0,5} \exp (i \varphi),
$$

where $R, \tau$ - equivalent resistance and time constant of the circuit at fig. 5 ,

$$
\begin{aligned}
& R=R_{i}\left(1+R_{i} R_{s}^{-1}\right)^{-1} ; \\
& \tau=C R ; \\
& C=C_{s}+C_{i} ; \\
& \varphi=-\operatorname{arctg}(\omega \tau) .
\end{aligned}
$$

From (16) transformation coefficient $K_{\mathrm{T}}$ is equal to:

$$
K_{T}=U J^{-1}=K_{s}\left(1+R_{i} R_{s}^{-1}\right)^{-1}\left(1+(\omega \tau)^{2}\right)^{-0,5} \exp (i \varphi)
$$

and

$$
K_{\mathrm{s}}=S R_{\mathrm{i}} \text {. }
$$

It is obvious to demand simultaneously two conditions:

$$
\left.\begin{array}{l}
R=R_{i} \\
(\omega \tau)^{2}=0
\end{array}\right\}
$$

for $K_{\mathrm{T}}$ be independent from the parameters of the medium. To this, $R_{\mathrm{i}}$ has to satisfy the relation

$$
R_{i}=n k T \Delta f\left(J_{N} S\right)^{-2},
$$

where $n, \Delta \mathrm{f}$ - noise factor and amplifier's bandwidth, $k$ - Boltzman's constant, $T$ - temperature, $J_{\mathrm{N}}$ - noise current density.

The parameters $f_{\mathrm{c}}$ - critical SLP frequency - and $f_{0}$ - quasiresonance SLP frequency - are introduced:

$$
\left.\begin{array}{l}
f_{c}=I_{s}\left(2 \pi \varepsilon_{0} K_{s}\right)^{-1} \\
f_{0}=\left(1+C_{i} C_{0}^{-1}\right)^{0,5} f_{p e}
\end{array}\right\}
$$


where $C_{0}$ is the sensor capacitance in vacuum, $f_{\mathrm{pe}}$ - Langmuir frequency of electrons in plasma, $\varepsilon_{0}$ - dielectric constant.

It is shown that the limits of frequency band where conditions (18) are accomplished are determined by the relation of $f_{\mathrm{c}}$ and $f_{\mathrm{pe}}$. The dependence of $K_{\mathrm{T}}$ on frequency for three different values $f_{\mathrm{pe} 1}<<f_{\mathrm{pe} 2}<<f_{\mathrm{pe} 3}$ is shown at fig. 6 .

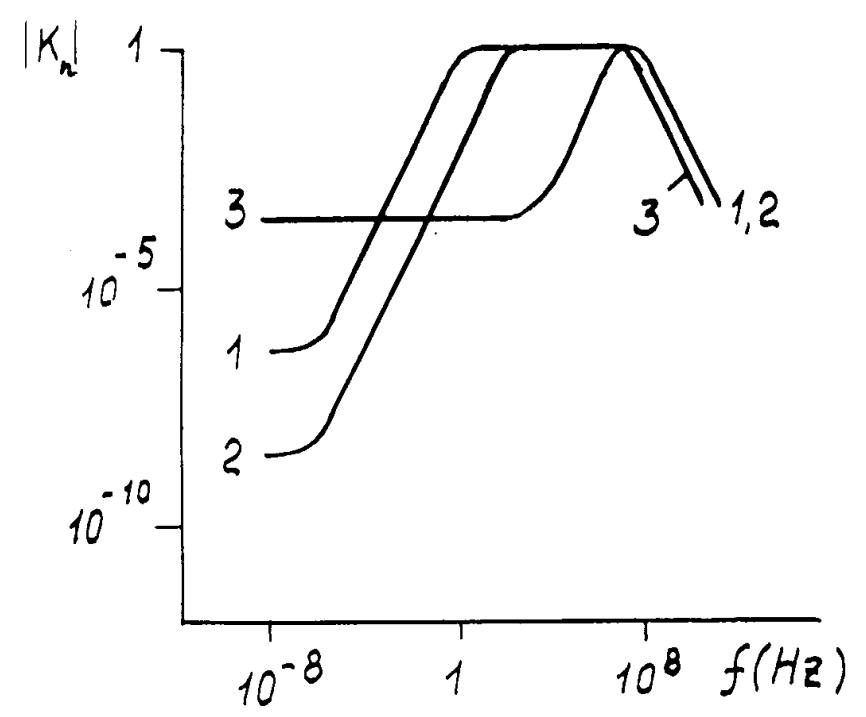

Fig.6. SLP transfer function

At quasi-resonance frequency $\left|K_{n}\right|=\left|K_{T} K_{s}^{-1}\right|$ has maximum value, equal to

$$
\left|K_{n}\right|_{\max }=\left(1+\mathrm{v}_{n} f_{c}^{-1}\left(1+C_{i} C_{0}^{-1}\right)\right)^{-1}
$$

where $\quad v_{n}=(2 \pi)^{-1} v, \quad v$ - electron-ion collision frequency. When $f \rightarrow \infty \quad\left|K_{n}\right| \rightarrow 0$; when $f \rightarrow 0$ $\left|K_{n}\right|=\left(1+\left(f_{c} v_{n}\right)^{-1} f_{p e}^{2}\right)^{-1}$. Our assumptions are valid for solar wind plasma (curves 1,2$)$ when $f \geq(1 \div 3) \times 10^{-2} \mathrm{~Hz}$ and for $F$-layer (curve 3) when $f \geq 5 \cdot 10^{3} \mathrm{~Hz}$.

It seems to be enough to prove that SLP really operates as electric current density to voltage transformer. The estimation of SLP noise level from (16), (19) gives the order of $10^{-13} \mathrm{~A} / \mathrm{cm}^{2}$ The most important noise source for SLP as for FC is sunlight which also can produce very dense flux of photoelectrons, masking measured current.

\section{CONCLUSION}

From the precedent description of three possible arrangements for spatial current density measurement it follows that all three of them can be used for this purpose. It simplicity the SLP has doubtless advantages. As to the external factors influence, both SLP and FC seem to be sensitive to the sunlight and all sensors are influenced by spacecraft potential. The last creates the potential barrier which does not allow to the charged particles with lower energies to reach the sensor.

These error sources have to be taken into account. But for low-altitude experiment (as it was mentioned, it is planned at around $600 \mathrm{~km}$ above the Earth) the charged particles concentration is much more high than this of the procured photoelectrons and their impact will be not significant if enough accurate frequency analysis of collected data will be done. Also the floating potential at this height is expected to be always negative and low enough. To this, the additional instrumentation onboard the satellite - magnetometers and electric sensors will give oblique data to compare with the directly measured values. 
It is expected that the planned comparative study will help to find the most reliable instrument for wave activity investigation in space plasma. These works were supported by grant INTAS 97-1769.

\section{REFERENCES}

Alleyne, H., M. Balikhin, J. Juchniewicz and J. Blecky, S. Klimov, V. Korepanov and F. Dudkin, G. Lizunov², F. Lefeuvre and V. Krasnoselskikh, Project «VARIANT»: Current and Field Measurements onboard SICH$1 \mathrm{M}$ Satellite, $32^{\text {nd }}$ Scientific Assembly of COSPAR, Nagoya, Japan, Abstracts (1998), p. 200.

Bering, E. A., M. C. Kelley, F. C. Mozer, and U.V. Fahleson., Theory and Operation of the split Langmuir Probe, Planet. Space Sci., 21, № 11 (1973), p. 1983-2001.

Korepanov, V. and R. Berkman, New Approach to the Exact Design of Low Noise Search-Coil Magnetometers, XIV IMEKO World Congress. New measurements - challenges and visions, Vol. IVA, Topic 4, Tampere, Finland (1997), p.p. 103-108.

Krasnosel'skikh, V., O. Randriamboarison, J.-P. Lebreton, F.-X. Sene, P. Fergeau, G. Avez, and M. Hamelin, Floating Potential of the Space Station and current density measurements around it, ESA (SP-385, December 1996), p.p. 347-351.

Safrankova, J., G. Zastenker, A. Fedorov, Z. Nemecek, M. Simersky, L. Prech, O. Vaisberg, Yu. Sharko, T. Romasenko, A. Leibov, M. Richter, T. Lesina, N. Plusnina and N. Yanovskaja,.Omnidirectional Plasma Sensor VDP, Interball. Mission and payload, CNES-IKI (1995), p.p. 195-198.

Vaisberg, O., S. Klimov and V. Korepanov, Current Density Measurements in the Shock Front by Means of Splitted Langmuir Probe, Soviet Journal Space Research, 27, № 3 (1989), p.p. 623-627. 